\title{
Experten-Evaluation von Web-GIS-Lösungen und Internetkartendiensten zur Kommunikation von Hochwasserinformationen
}

\author{
Maria Hagemeier-Klose, Klaus Wagner, Freising
}

\section{Einleitung}

Das Internet befindet sich auf dem Weg zum «Geospatial Web» und zur vermehrten «Geo-Kommunikation» (Möller 2006; Scharl \& Tochtermann 2007). Man denke hier an die Verbreitung von Google Earth, Google Maps und anderen geographischen Informationsdiensten im Internet. Nun gehen auch die staatlichen Behörden vermehrt dazu über, die Informationen über Naturgefahren bzw. -risiken kartographisch durch Geoinformationssyteme (GIS) oder Internetkartendienste im Internet zur Verfügung zu stellen. Grundsätzlich können dabei vier Typen von Kartenwerken unterschieden werden:

- Kataster, die abgelaufene Ereignisse dokumentieren, z.B. Lawinenkataster.

- Karten, die die Ausdehnung eines natürlichen Prozesses für eine oder mehrere Eintrittswahrscheinlichkeiten bzw. Wiederkehrperioden verdeutlichen, z.B. Überschwemmungsgebiete in Deutschland.

- Karten, die sowohl Magnitude als auch Frequenz der natürlichen Prozesse berücksichtigen, z.B. Gefahrenkarten in Österreich und der Schweiz.

- Karten, die auch das Schadenspotenzial berücksichtigen, z.B. die Hochwasserrisikokarten entsprechend der EU Richtlinie 2007/60/EG über die Bewertung und das Management von Hochwasserrisiken.

Im Folgenden wird der Begriff der Gefahrenkarten als Überbegriff über alle hier genannten Typen verwendet. Durch die rechtliche Codierung solcher Kartenwerke kann einerseits die Bauentwicklung beschränkt werden, andererseits dienen die Gefahrenkarten der Information der Bevölkerung. In Europa ist die Wasserwirtschaft durch die EU-Hochwasserschutz-Direktive angehalten, Hochwassergefahrenkarten einzuführen bzw. weiterzuentwickeln und dabei die Öffentlichkeit zu informieren (Bundesministerium FÜr UMWELT, NATURSCHUTZ UND ReAKtorsicherheIt, BMU, 2007). Die in diesem Beitrag analysierten Staaten und Länder haben alle bereits begonnen, Gefahrenkarten zu erstellen und z.T. auch im Internet zur Verfügung zu stellen. Ob das Ziel, die Bevölkerung durch aktuelle Kartenwerke zu informieren, derzeit erreicht wird, darf anhand der Befragungsergebnisse aus Österreich und der Schweiz bezweifelt werden, in denen die Befragten ihr persönliches Risiko durch Naturgefahren deutlich unterschätzen (NATIONALE PlatTFORM NATURGEFAhren, PLANAT, 2004; Schrems 1998; SiEgrist \& GUTSCHER 2006).
Eine Hürde für die erfolgreiche Internetkommunikation ist die Internetnutzung durch die betroffene Bevölkerung. Sowohl in der Schweiz als auch in Deutschland hat der Anteil der Internetnutzer zwischen 1997 und 2007 stark zugenommen:

- In der Schweiz von 15\% auf 76\% (BUNDESAMT FÜR STATISTIK, BFS, 2007),

- in Deutschland von 7\% auf 63\% (VAN EIMEREN \& FREES 2007).

Der Anteil der Personen, die (fast) täglich das Internet nutzen, liegt in der Schweiz bei 51\% (BFS 2007) und in Deutschland bei 42\% (ARBEITSGEMEINSCHAFT ONLINE Forschung, AGOF, 2007). In Deutschland nutzen 32\% (EU 26\%) der Bevölkerung das Internet gelegentlich für die Interaktion mit staatlichen Behörden (EUROSTAT 2007). Im Verhältnis zu den Massenmedien und dem Gespräch mit Nachbarn spielte in der Studie von WAGNER (2004) das Internet zur Informationsgewinnung über Naturgefahren noch eine untergeordnete Rolle. Trotzdem dürfte in Zukunft das Internet gerade für jüngere und mobilere Bürger, die weniger in die lokalen Kommunikationszirkel eingebunden sind, einen wichtigeren Platz in der Information über die lokale Bedrohungssituation einnehmen.

Ziel dieses Beitrags ist es, einen Überblick über die im Internet verfügbaren Kartendienste bzw. GIS-Lösungen zu bieten. Dabei wird nicht nur analysiert, welche Arten von Informationen zur Verfügung gestellt werden, sondern auch, inwieweit die dargestellten Informationen für die Nutzergruppe der breiten Bevölkerung verständlich und nachvollziehbar sind. Diese Analyse erfolgte dabei nicht durch eine quantitative Befragung potentieller Nutzer, sondern aus Sicht von Experten mittels einer formativen Evaluation (WotTaWA \& Thierau 2003). Im folgenden Kapitel werden die Vorgehensweise und das entwickelte Evaluationsschema vorgestellt, das danach beispielhaft an vier sich in grundlegenden Variablen unterscheidenden Kartendiensten bzw. Web-GIS verdeutlicht wird. Empfehlungen für die Fortentwicklung von Internetangeboten schließen den Beitrag ab.

\section{Methodik}

Die Analyse der im Internet verfügbaren Kartendienste und GIS-Lösungen wurde innerhalb des Teilprojekts Kommunikation und Information des durch das EU Life-Programm geförderten Projekts Floodscan 


\begin{tabular}{|c|c|c|c|c|}
\hline & Nationale Ebene & $\begin{array}{l}\text { Länder-/Kantons-/ } \\
\text { Provinzebene }\end{array}$ & $\begin{array}{l}\text { Flussgebiets-/ } \\
\text { regionale Ebene }\end{array}$ & $\begin{array}{l}\text { Lokale Ebene: } \\
\text { Städte, Landkreise }\end{array}$ \\
\hline $\begin{array}{l}\text { Komplexes Raum- } \\
\text { Informations-System } \\
\text { GIS-Funktionalität, } \\
\text { Darstellung einer oder } \\
\text { mehrerer Naturgefahren }\end{array}$ & & $\begin{array}{l}\text { - Kärnten Atlas } \\
\text { - Salzburger GIS } \\
\text { - Geoportal Rheinland } \\
\text { Pfalz } \\
\text { - GIS Kanton Zürich }\end{array}$ & & \\
\hline $\begin{array}{l}\text { Komplexes } \\
\text { Informations-System } \\
\text { Darstellung mehrerer } \\
\text { Naturgefahren }\end{array}$ & & & $\begin{array}{l}\text { - Informations- } \\
\text { dienst Alpine } \\
\text { Naturgefahren } \\
\text { (IAN) }\end{array}$ & \\
\hline $\begin{array}{l}\text { Komplexes } \\
\text { Informations-System } \\
\text { Darstellung einer } \\
\text { Naturgefahr }\end{array}$ & $\begin{array}{l}\text { eHORA - } \\
\text { Hochwasser- } \\
\text { risiko } \\
\text { Österreich }\end{array}$ & $\begin{array}{l}\text { - Saarland GIS } \\
\text { Überschwemmungs- } \\
\text { gebiete }\end{array}$ & & \\
\hline $\begin{array}{l}\text { Kartendienst } \\
\text { Darstellung mehrerer } \\
\text { Naturgefahren }\end{array}$ & $\begin{array}{l}\text { - Risikokarte } \\
\text { Niederlande }\end{array}$ & $\begin{array}{l}\text { - Gefahrenhinweis- } \\
\text { karte Luzern } \\
\text { - Risikokarte } \\
\text { Flevoland, NL }\end{array}$ & & \\
\hline $\begin{array}{l}\text { Kartendienst } \\
\text { mit Darstellung einer } \\
\text { Naturgefahr }\end{array}$ & - Flood Map GB & $\begin{array}{l}\text { - Informationsdienst } \\
\text { Überschwem- } \\
\text { mungsgefährdete } \\
\text { Gebiete in Bayern } \\
\text { (IÜG) } \\
\text { - Hochwasser- } \\
\text { gefahrenkarten } \\
\text { Sachsen }\end{array}$ & $\begin{array}{l}\text { - Hochwasser- } \\
\text { gefahrenkarte } \\
\text { Baden- } \\
\text { Württemberg } \\
\text { - Mosel } \\
\text { Gefahrenatlas }\end{array}$ & $\begin{array}{l}\text { Hochwasser- } \\
\text { gefahrenkarten } \\
\text { Köln } \\
\text { - Landkreis } \\
\text { Schwäbisch-Hall }\end{array}$ \\
\hline
\end{tabular}

Tab. 1:Typologie von aktuellen Kartendiensten und Web-GIS-Lösungen; die im Beitrag näher analysierten Beispiele sind fett gedruckt.

Typology of current web mapping services and WebGIS solutions; the examples analysed in the text are written in bold. Typologie des services cartographiques actuels et des solutions Web-SIG; les exemples analysés dans le texte figurent en gras.

Quelle: eigene Darstellung

durchgeführt. Das Teilprojekt analysiert und optimiert die Darstellung unterschiedlicher Gefahrenkarten sowie deren Umsetzung als Web-Mapping-Service oder Web-GIS im Internet mit Hilfe formativer und summativer Evaluation. Dieser Beitrag dokumentiert, analysiert und präsentiert aktuelle Ergebnisse der formativen Evaluation, die mittels der drei Arbeitsschritte Typisierung, Kriterienüberprüfung und Experten-Bewertung umgesetzt wurde.

\subsection{Internetrecherche und Typisierung der Auftritte}

Eine Ist-Analyse (durchgeführt Januar-März 2007) dokumentiert einen Überblick über in der Praxis eingesetzte Kartendienste und Web-GIS zur Information der Bevölkerung über Hochwasser. Recherchiert wurde in Deutschland, Österreich, der Schweiz, den
Niederlanden sowie Großbritannien. Da sich diese Analyse um Angebote im Medium Internet dreht, wurde die Recherche nach einem kurzen Literaturreview weitgehend im Internet durchgeführt. Die Autoren analysierten zunächst offizielle Internetseiten von relevanten Behörden, auf welchen sie die meisten Ergebnisse erzielten. Als letzter Rechercheschritt wurde mit Hilfe verschiedener Begriffe (z.B. Kartendienst + Hochwasser, GIS + Hochwasser, Gefahrenkarten) eine Suchmaschinen-Recherche in Google durchgeführt. Mit dieser Methode konnten noch drei, zuvor noch nicht bekannte Angebote gefunden werden.

Die wichtigsten Kartendienste und GIS-Lösungen wurden anhand der Kriterien Maßstab/Raumbezug und Komplexität typisiert. Tabelle 1 zeigt die Ergeb- 
nisse dieser Typisierung und hebt die Angebote in Fettschrift hervor, welche im Ergebnisteil beispielhaft einer weitergehenden Evaluation unterzogen werden. Die vier vertieften Beispiele repräsentieren sowohl die unterschiedlichen Formen der Darstellung als auch der Komplexitätsgrade und räumlichen Bezugsebenen. Die Auswahl von nur deutschsprachigen Angeboten zur vertieften Analyse erfolgte aus forschungspraktischen Überlegungen - die Evaluatoren waren alle deutsche Muttersprachler. Andererseits repräsentieren die ausgewählten deutschsprachigen Dienste alle wesentlichen Merkmale der Informationsangebote, die in den analysierten europäischen Ländern vorzufinden sind.

Während zwischen der nationalen und Länderebene kein systematischer Unterschied offensichtlich ist, ist auf lokaler Ebene eine stärkere Nutzeranpassung möglich (Gefahrenkarten Köln). Die bayerischen Angebote wurden aufgrund des Projektzusammenhangs ausgewählt, der Kärnten Atlas aufgrund seiner hohen Komplexität und überregionalen Ausrichtung.

\subsection{Evaluationsschema}

Das zur Bewertung der Internetangebote angewandte Evaluationsschema wurde aus den Ansätzen verschiedener Autoren entwickelt und gliedert sich dabei in drei Dimensionen (Alexander \& Tate 1999; DeutSCHe Bundesstiftung UmWelt, DBU, 2005; Riemer \& Müller-LanKenau 2005; SMITH 1997):

- Inhaltliche Dimension: Textumfang/-qualität, Aktualität, Informationsgehalt, weiterführende Informationen

- Rhetorisch-kommunikative Dimension: Zielgruppenorientierung, Autorenschaft, Glaubwürdigkeit, Zielsetzungen, Verständlichkeit

- Multimedial-funktionale Dimension: Funktionalität, Gestaltung, Systemanforderungen, Interaktivität.

Bei der Bewertung der inhaltlichen Dimension geht es darum, ob die Inhalte der Internetangebote geeignet sind, die Bevölkerung über Hochwasser zu informieren. Bei Kartenwerken geht es zum einen um direkt in den Karten dargestellte Inhalte, aber auch um die Bereitstellung von Zusatzmaterial. Nicht nur Quantität, sondern auch Qualität sind hier wichtige Evaluationskriterien. Da das Internet quasi in Echtzeit operiert, ist zudem die Aktualität der Inhalte von besonderer Bedeutung.

Die rhetorisch-kommunikative Dimension dient zur Analyse der Akzeptanz der angebotenen Informationen, der Zielsetzung der Angebote sowie der Überprüfung der Zielgruppenorientierung. Gerade im Internet sind Transparenz, Autorenschaft und Glaubwürdigkeit wichtige Qualitätsmerkmale, da bei vielen Internet- angeboten die Quellen der Informationen nicht klar ersichtlich sind, was sich negativ auf die Glaubwürdigkeit auswirken kann.

Im multimedial-funktionalen Bereich geht es darum, optische Gestaltung, Anwendbarkeit, Interaktivität und Funktionalität zu evaluieren. Gerade bei GIS oder Kartendiensten bildet die Funktionalität eines der wichtigsten Kriterien, wobei nicht nur Vielfalt, sondern auch Verständlichkeit von Funktionen, Navigation und Orientierung von Bedeutung sind. Die Interaktionsmöglichkeiten für die Nutzer z.B. über Foren, Feedback-E-Mails oder Datenexport ist bei Internetangeboten ebenfalls zentral. Eine übersichtliche und ansprechende Darstellung ist hier desgleichen ein wichtiges Kriterium.

Da sich die vorliegende Evaluation auf die Zielgruppe der breiten Bevölkerung bezieht, wird im Wesentlichen auf die für diese Nutzergruppe relevanten Kriterien aus den genannten Dimensionen eingegangen.

\subsection{Expertenworkshop}

Die zur Evaluation ausgewählten Angebote wurden an einem interdisziplinären Workshop von Experten aus den Bereichen Wasserwirtschaft, Wasserbau, Geoinformatik, Kommunikationswissenschaft und öffentlicher Verwaltung in einer Gruppendiskussion analysiert. Dabei wurden auch Anforderungen und Empfehlungen zur Realisierung von Internetkartendiensten bzw. GIS-Lösungen entwickelt.

\section{Ergebnisse der Evaluation}

Die ausführliche Darstellung der Evaluationsergebnisse erfolgt in den Tabellen 2-4. Die Ergebnisse leiten sich zum einen aus dem Expertenworkshop, zum anderen aus unabhängigen Anwendungstests der Autoren ab. Aufgrund dieser qualitativ orientierten Methodik setzen die Autoren im Wesentlichen auch qualitative Kriterien zur Bewertung der Informationsangebote ein. Für eine bessere Übersichtlichkeit der Darstellung der Ergebnisse wird die Ausprägung der Kriterien in positive (hellgrau unterlegte Zellen), neutrale (grau unterlegte Zellen) und negative (dunkelgrau unterlegte Zellen) zusammengefasst.

\subsection{Inhaltliche Dimension}

Die Evaluation der inhaltlichen Merkmale (Tab. 2) soll hier vergleichend nur für das Themengebiet Hochwasser vorgestellt werden. Der Kärnten Atlas verdeutlicht auf der Basis einer topographischen Karte oder eines schwarz-weißen Luftbilds drei Gefahrenzonen (Hochrisikozone, Restrisikozone, Zone ohne Hochwasserrisiken). Daneben werden Anschlagslinien von Hochwasserereignissen für HQ 30 und HQ 100 aufge- 


\begin{tabular}{|c|c|c|c|c|}
\hline $\begin{array}{l}\text { Evaluations- } \\
\text { kriterien }\end{array}$ & Kärnten Atlas & IAN & İ̈G & $\begin{array}{l}\text { Gefahrenkarten } \\
\text { Köln } \\
\end{array}$ \\
\hline \multicolumn{5}{|c|}{ Inhaltliche Dimension } \\
\hline Basis-Karten & $\begin{array}{l}\text { - S/W-Luftbild } \\
\text { - Kataster } \\
\text { - ÖK } 50\end{array}$ & $\begin{array}{l}\text { - Orthofoto } \\
\text { - TK } 25\end{array}$ & $\begin{array}{l}\text { - Flurkarte } \\
\text { - TK } 25\end{array}$ & - Stadtplan \\
\hline Themen/Layer & $\begin{array}{l}\text { - Vielfältige } \\
\text { räumliche Themen } \\
\text { - Anschlagslinien } \\
\text { von Hochwasser- } \\
\text { ereignissen } \\
\text { verschiedener } \\
\text { Jährlichkeit } \\
\text { (HQ 30, HQ 100) } \\
\text { - Raumordnerische } \\
\text { Gefahrenzonen }\end{array}$ & 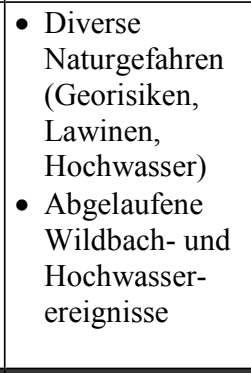 & $\begin{array}{l}\text { - Wassersensibler } \\
\text { Bereich (Gegenden } \\
\text { mit Bodentypen, } \\
\text { die Grund- oder } \\
\text { Hochwasserein- } \\
\text { fluss anzeigen) } \\
\text { - Rechtlich fest- } \\
\text { gesetzte und ermit- } \\
\text { telte Überschwem- } \\
\text { mungsgebiete } \\
\end{array}$ & $\begin{array}{l}\text { - Hochwasserflächen } \\
\text { für verschiedene } \\
\text { Pegelstände des } \\
\text { Rheins mit } \\
\text { zugehörigen } \\
\text { Überflutungstiefen, } \\
\text { mit und ohne } \\
\text { Berücksichtigung } \\
\text { technischer } \\
\text { Schutzmaßnahmen } \\
\end{array}$ \\
\hline $\begin{array}{l}\text { Zusatz- } \\
\text { informationen } \\
\text { (Quantität und } \\
\text { Qualität) }\end{array}$ & $\begin{array}{l}\text { Wenig Zusatz- } \\
\text { information zum } \\
\text { Thema Hochwasser } \\
\text { - Vorstellung der } \\
\text { Zielsetzung } \\
\text { - Downloads von } \\
\text { Publikationen, } \\
\text { Flyern } \\
\text { - Links, } \\
\text { Ansprechpartner }\end{array}$ & $\begin{array}{l}\text { Keine Zusatz- } \\
\text { information, nur } \\
\text { Kurzbeschreibung } \\
\text { vorhanden }\end{array}$ & $\begin{array}{l}\text { Gut aufbereitetes, } \\
\text { breites Zusatzmaterial } \\
\text { - Nutzungs- } \\
\text { beschränkungen } \\
\text { - Festsetzungs- } \\
\text { verfahren } \\
\text { - Gegenmaßnahmen } \\
\text { - rechtl. Hinweise } \\
\text { - Glossar, Links, } \\
\text { Ansprechpartner }\end{array}$ & $\begin{array}{l}\text { Breite Zusatz- } \\
\text { information } \\
\text { - Hochwasserschutz } \\
\text { - Risikomanagement } \\
\text { - Ansprechpartner } \\
\text { - Aktueller } \\
\text { Pegelstand }\end{array}$ \\
\hline Aktualität & Nicht erkennbar & November 2006 & 31.08 .2007 & Nicht erkennbar \\
\hline
\end{tabular}

Tab. 2: Experten-Evaluation der Internet-Kartendienste und Web-GIS: inhaltliche Dimension Expert evaluation of web mapping services and WebGIS: content

Evaluation par des experts des services cartographiques web et Web-SIG: contenu

Quelle: eigene Darstellung

zeichnet. Die letzte Aktualisierung der Informationen ist nicht ersichtlich.

Der Informationsdienst Alpine Naturgefahren (IAN) stellt auf einer topographischen Karte oder einem Luftbild abgelaufene Wildbach- und Hochwasserereignisse dar, zu denen die entsprechenden Datenblätter mittels Hyperlink abgerufen werden können, letztmalig aktualisiert im November 2006.

Der Informationsdienst Überschwemmungsgefährdete Gebiete in Bayern (IÜG) zeigt die Ausdehnung der amtlich festgesetzten und ermittelten Überschwemmungsgebiete, denen in der Regel das hundertjährliche Hochwasser zu Grunde gelegt wird. Darüber hinaus wird die Ausdehnung des so genannten wassersensiblen Bereiches in einem separaten Layer aufgezeichnet, in dem aufgrund der Bodentypen auf eine historische bzw. gegenwärtige Beeinflussung durch Grund- oder Hochwasser geschlossen werden kann. Für die Überschwemmungsgebiete wird in der höchsten Zoom-Stufe die digitale Flurkarte angezeigt, womit eine sehr viel höhere Auflösung als in vergleichbaren Internetkartendiensten angeboten wird. In niedrigeren Zoomstufen wird hier als Grundlage die topographische Karte angezeigt. Die dargestellten Informationen wurden im August 2007 letztmalig aktualisiert.

Die Hochwassergefahrenkarten Köln zeigen die Überschwemmungstiefen und -ausbreitungen bei verschiedenen Rheinwasserständen am Kölner Pegel mit und ohne Berücksichtigung geplanter Hochwasserschutzanlagen, wobei der Kölner Stadtplan als Basiskarte dient. $\mathrm{Ob}$ die gezeigten Daten aktuell sind, ist nicht ersichtlich. Als Zusatzinformation können Fotos von 


\begin{tabular}{|c|c|c|c|c|}
\hline $\begin{array}{l}\text { Evaluations- } \\
\text { kriterien }\end{array}$ & Kärnten Atlas & IAN & IÜG & $\begin{array}{l}\text { Gefahrenkarten } \\
\text { Köln } \\
\end{array}$ \\
\hline \multicolumn{5}{|c|}{ Rhetorisch-kommunikative Dimension } \\
\hline $\begin{array}{l}\text { Autorenschaft, } \\
\text { Inhaltsquelle }\end{array}$ & $\begin{array}{l}\text { Amt der Kärnter } \\
\text { Landesregierung, } \\
\text { Landesplanung }\end{array}$ & $\begin{array}{l}\text { Bayerisches } \\
\text { Landesamt für } \\
\text { Umwelt (LfU) } \\
\end{array}$ & $\begin{array}{l}\text { Bayerisches } \\
\text { Landesamt für } \\
\text { Umwelt (LfU) } \\
\end{array}$ & $\begin{array}{l}\text { Stadtentwässerungs- } \\
\text { betriebe Köln (STEB) }\end{array}$ \\
\hline $\begin{array}{l}\text { Glaub- } \\
\text { würdigkeit }\end{array}$ & - Behörde & - Behörde & - Behörde & - Gemeinde \\
\hline Zielsetzung & $\begin{array}{l}\text { - Erfassung, } \\
\text { Verwaltung und } \\
\text { Bereitstellung } \\
\text { umfassender } \\
\text { Geodaten und } \\
\text { Geoinformationen } \\
\text { - Informationen für } \\
\text { Bürger/Innen, } \\
\text { Unternehmen und } \\
\text { Verwaltungen }\end{array}$ & $\begin{array}{l}\text { - Information von } \\
\text { Bürger/Innen, } \\
\text { Gemeinden, } \\
\text { Planern } \\
\text { - Verbesserung der } \\
\text { Vorsorge } \\
\text { - Erkennen von } \\
\text { Risiken } \\
\text { - Bewusstseins- } \\
\text { stärkung }\end{array}$ & $\begin{array}{l}\text { - Information von } \\
\text { Bürger/Innen, } \\
\text { Gemeinden, } \\
\text { Planern } \\
\text { - Verbesserung der } \\
\text { Vorsorge } \\
\text { - Erkennen von } \\
\text { Risiken } \\
\text { - Stärkung des } \\
\text { Bewusstseins } \\
\end{array}$ & $\begin{array}{l}\text { - Jeder Interessierte } \\
\text { kann sich } \\
\text { informieren, wie } \\
\text { hochwasser- } \\
\text { betroffen sein } \\
\text { Grundstück oder } \\
\text { Stadtteil ist. }\end{array}$ \\
\hline Zielgruppe(n) & $\begin{array}{l}\text { - Öffentlichkeit } \\
\text { - Unternehmen } \\
\text { - Verwaltungen }\end{array}$ & $\begin{array}{l}\text { - Öffentlichkeit } \\
\text { - Gemeinden, } \\
\text { Verwaltungen, } \\
\text { Planer }\end{array}$ & $\begin{array}{l}\text { - Öffentlichkeit } \\
\text { - Gemeinden, } \\
\text { Verwaltungen, } \\
\text { Planer }\end{array}$ & - Jeder Interessierte \\
\hline $\begin{array}{l}\text { Verständlichkeit } \\
\text { (Ausrichtung } \\
\text { auf Ziel- } \\
\text { gruppe(n)) }\end{array}$ & $\begin{array}{l}\text { - Sehr komplex } \\
\text { - Themenauswahl } \\
\text { für Laien schwierig } \\
\text { - Eher für } \\
\text { Fachanwender } \\
\text { geeignet }\end{array}$ & $\begin{array}{l}\text { - Sehr komplex } \\
\text { - Themenauswahl } \\
\text { für Laien schwierig } \\
\text { - Eher für } \\
\text { Fachanwender } \\
\text { geeignet }\end{array}$ & $\begin{array}{l}\text { - Einfach gehalten, } \\
\text { auch für Laien } \\
\text { verständlich } \\
\text { - Wenig Funktionen } \\
\text { bzw. Daten für } \\
\text { Fachanwender } \\
\end{array}$ & $\begin{array}{l}\text { - Einfach gehalten, } \\
\text { auch für Laien } \\
\text { verständlich } \\
\text { - Wenig Funktionen } \\
\text { bzw. Daten für } \\
\text { Fachanwender } \\
\end{array}$ \\
\hline
\end{tabular}

Tab. 3: Experten-Evaluation der Internet-Kartendienste und Web-GIS: rhetorisch-kommunikative Dimension Expert evaluation of web mapping services and WebGIS: rhetoric and communication Evaluation par des experts des services cartographiques web et Web-SIG: rhétorique et communication Quelle: eigene Darstellung

vergangenen Hochwasserereignissen über Hyperlinks in der Karte angezeigt werden.

Die beiden erstgenannten Angebote bieten im Gegensatz zum IÜG keine über den Kartendienst hinausgehenden Informationen zur Hochwassergefahr, zur Art der Ermittlung der vorgestellten Situation, zu Rechtsfolgen oder möglichen Schutzmaßnahmen. Die Hochwassergefahrenkarten Köln sind in den Internetauftritt der Hochwasserschutzzentrale eingebunden, so dass dem Nutzer darüber hinaus weitere hochwasserrelevante Informationen zur Verfügung stehen.

\subsection{Rhetorisch-kommunikative Dimension}

Alle evaluierten Internetangebote sind von Behörden konzipiert, weshalb die Reputation bzw. die Glaub- würdigkeit der bereitgestellten Informationen als hoch eingestuft werden kann (siehe Tab. 3). Es bestehen keine Nutzungseinschränkungen, Registrierungszwänge oder Kosten bei den Diensten. Die Zielsetzung der vorliegenden Seiten umfasst die Information der Bevölkerung, von Unternehmen, Planern und Verwaltungen, welche auch explizit als Zielgruppen der Angebote genannt werden. Bezüglich der Zielgruppenausrichtung sahen die Experten den Kärnten Atlas als geeignetes Tool für Fachanwender und Schulen an, da Verknüpfungen unterschiedlichster Themen möglich sind. Der IAN ist wie der Kärnten Atlas für GIS-Laien zu komplex und daher eher ein Informationstool für Fachanwender. Der IÜG und das Kölner Angebot wurden als geeignet für die breite Bevölkerung, jedoch als zu einfach für Fachanwender bewertet. 


\subsection{Multimedial-funktionale Dimension}

Tabelle 4 zeigt eine ausführliche Übersicht über die Bewertungsergebnisse innerhalb der multimedialfunktionalen Evaluationsdimension. Die Interaktionsmöglichkeiten sind stark eingeschränkt, da die Nutzer keine Möglichkeit haben, die Seiten durch eigene Daten, Dokumente, Bilder oder Ähnliches aktiv zu gestalten oder Anmerkungen und Beurteilungen z.B. in Foren zu platzieren. Auch ist es nicht möglich, Suchergebnisse zu speichern oder individuelle Markierungen zu setzen. Die Nutzerinteraktivität beschränkt sich auf Kartenerstellung, Ausdrucken oder Versenden bestimmter Kartenausschnitte, oder die Suche z.B. nach bestimmten Orten, Wohngebieten, Adressen. Ebenso sind bei allen analysierten Angeboten keine Verbindungen zu Echtzeitinformationen wie Pegelständen oder Web-Cams und keine 3D-Darstellungen vorhanden.

Bezüglich der Funktionalität vereinigt der Kärnten Atlas alle gängigen GIS-Funktionen wie $\mathrm{Zu}$ - oder Abschalten verschiedener Inhaltslayer und Basiskarten, ausgeweitete Zoom-, Abfrage-, Such- und Messfunktionen, Objektdatenanzeige, Hyperlinks und Legendenanzeige. Diese umfassende Umsetzung als Web-GIS wurde positiv evaluiert, da sie besonders für Spezialisten viele verschiedene räumliche Informationen und die komplexe GIS-Funktionalität bereitstellt. Gerade die Komplexität und die Vielfalt an diversen Informationen wurden aber auch als problematisch angesehen, da besonders Nicht-Fachleute hiermit überfordert sein könnten. Die Legende wurde aufgrund zu weniger Informationen und fehlender Erklärungen bemängelt.

In der Funktionalität gleicht der IAN als Web-GIS dem Kärnten Atlas, bietet aber im Mess- und Abfragebereich weniger Möglichkeiten. Im Bereich Hochwasser stellt er ein Kataster für abgelaufene Ereignisse dar und gibt keine Auskunft über gegenwärtige und zukünftige Hochwassergefahren. Auch die optische Gestaltung wurde als unübersichtlich und ungeeignet zur Kommunikation mit der Bevölkerung angesehen.

Funktional handelt es sich beim IÜG um einen nur eingeschränkt interaktiven Kartendienst. Er bietet eine große Auswahl an Suchfunktionen, eine Druckfunktion und Zoomfunktionen, die jedoch nur feste Zoomausschnitte zeigen. Gerade die ausgeweitete Suchfunktionalität wurde positiv bewertet, ebenso wie die Zoomfunktionalität bis zur digitalen Flurkarte. Über einen Informationsknopf können Zusatzinformationen abgerufen werden, die eher knapp gehalten sind. Die einfache Handhabung und die Verbindung mit Texten, Erklärungen und Hintergrundinformationen fanden sehr positiven Anklang. Als unpassend empfanden die Bewerter die farbliche Darstellung - rote und gelbe Farben werden nicht direkt mit Hochwasser assoziiert. Die Texte seien z.T. zu umfangreich für das Internet und die Funktionalität und das Design des Kartendienstes zu statisch und zu wenig ansprechend. Zudem fehlten dem Angebot alternative Layer, z.B. mit der Darstellung von Wassertiefen, Fließgeschwindigkeiten oder Hochwasserereignissen verschiedener Jährlichkeit. Zudem sei der IÜG ungenügend verlinkt, beispielsweise mit Angeboten wie Hochwasserwarndiensten.

Die Funktionalität bei den Hochwassergefahrenkarten Köln ist auf einfache Kartendienstfunktionen beschränkt. Auch bei diesem Angebot wurden sowohl positive als auch negative Evaluationsaspekte ausgemacht. Die Möglichkeit, über in der Karte platzierte «Hotlinks» Fotos von vergangenen Hochwasserereignissen und Hintergrundinformationen zur Hochwassersituation abzurufen, fand bei den Evaluatoren großen Anklang. Das in den Kartendienst integrierte Symbol einer Pegellatte sei augenfällig und rufe in den Nutzern Assoziationen zu Hochwasser hervor. Gute Suchfunktionalität, dynamische, interaktive Kartendarstellung und die Kombination mit Pegelständen wurden ebenfalls positiv herausgestellt. Die Farbgebung der Karten - die Wassertiefen werden mit den Farben blau, rot, grün und gelb wiedergegeben, wobei gelb eine geringere Überschwemmungstiefe anzeigt als grün - wurde als unpassend empfunden. Zudem wurden die fehlende Übersichtlichkeit sowie die schwer zu findende Legende bemängelt.

Keiner der näher analysierten Informationsdienste kann optisch als besonders positiv herausgestellt werden, wobei die Gesamtgestaltung, von der Farbwahl abgesehen, der Kölner Gefahrenkarten am besten evaluiert wurde. Ein Negativbeispiel bezüglich der Gesamtgestaltung ist der Informationsdienst Alpine Naturgefahren.

\section{Diskussion und Empfehlungen: nutzerangepasste Internetangebote}

Ein entscheidendes Problem besteht in der Komplexität und in der Kartenlesbarkeit von geographischen Informationsangeboten zum Thema Hochwasser. Werden $\mathrm{zu}$ viele Informationen in eine Karte aufgenommen, so leiden die Verständlichkeit und Anwendbarkeit, insbesondere für die Bevölkerung. Beschränkt man die dargestellten Informationen, so fehlen Fachanwendern oder auch fachlich versierten Nutzern aus der Bevölkerung wichtige Informationen. Zuviel Funktionalität wirkt auf Laien eher verwirrend und abschreckend, zu wenig Funktionalität ist aber anderen Anwendern nicht ausreichend. Auch fehlendes Kartenmaterial oder fehlende Anwendungsoptionen 


\begin{tabular}{|c|c|c|c|c|}
\hline \begin{tabular}{|l}
$\begin{array}{l}\text { Evaluations- } \\
\text { kriterien }\end{array}$ \\
\end{tabular} & Kärnten Atlas & IAN & IÜG & $\begin{array}{l}\text { Gefahrenkarten } \\
\text { Köln }\end{array}$ \\
\hline \multicolumn{5}{|c|}{ Multimedial-funktionale Dimension } \\
\hline Funktionalität & - GIS-Funktionalität & - GIS-Funktionalität & - Kartendienst & - Kartendienst \\
\hline $\begin{array}{l}\text { Übersichtskarte mit } \\
\text { Navigationsfunktion }\end{array}$ & Vorhanden & Vorhanden & Vorhanden & Vorhanden \\
\hline Zoomfunktion & Freier Zoom & Freier Zoom & Feste Zoomstufen & Zoomstufen \\
\hline Messfunktionen & Strecke, Fläche & Strecken & Keine & Strecken \\
\hline $\begin{array}{l}\text { Objektdatenanzeige, } \\
\text { Info-Knopf }\end{array}$ & Vorhanden & Vorhanden & $\begin{array}{l}\text { Vorhanden, wenig } \\
\text { Information }\end{array}$ & Keine \\
\hline Suchfunktionen & Gute Suchoptionen & $\begin{array}{l}\text { Eingeschränkte } \\
\text { Suchoptionen }\end{array}$ & $\begin{array}{l}\text { Sehr gute, vielfältige } \\
\text { Suchoptionen }\end{array}$ & Gute Suchoptionen \\
\hline Layer zu/-abschalten & Vorhanden & Vorhanden & Vorhanden & Vorhanden \\
\hline Legende & $\begin{array}{l}\text { Vorhanden, zu wenig } \\
\text { Informationen }\end{array}$ & Vorhanden & Vorhanden & $\begin{array}{l}\text { Vorhanden, aber } \\
\text { versteckt }\end{array}$ \\
\hline Hyperlinks & $\begin{array}{l}\text { Vorhanden, aber nur } \\
\text { bei bestimmten } \\
\text { Themen nutzbar }\end{array}$ & $\begin{array}{l}\text { Vorhanden, aber nur } \\
\text { bei bestimmten } \\
\text { Themen nutzbar }\end{array}$ & Keine & $\begin{array}{l}\text { Vorhanden, Bilder } \\
\text { historischer Ereig- } \\
\text { nisse }\end{array}$ \\
\hline Druck/Export & $\begin{array}{l}\text { Vorhanden, Export } \\
\text { in Google Earth, } \\
\text { PDF }\end{array}$ & Vorhanden & Vorhanden & Vorhanden \\
\hline Hilfe & Vorhanden & Vorhanden & Vorhanden & Vorhanden \\
\hline \begin{tabular}{|l|} 
Interaktion/ \\
Kommunikation \\
\end{tabular} & $\begin{array}{l}\text { - Feedback } \\
\text { - Daten-Download }\end{array}$ & $\begin{array}{l}\text { - Download von } \\
\text { Metadaten }\end{array}$ & $\begin{array}{l}\text { - E-Mail an } \\
\text { Ansprechpartner }\end{array}$ & Keine \\
\hline Darstellung & $\begin{array}{l}\text { Zu komplex, } \\
\text { Kartenlesbarkeit } \\
\text { eingeschränkt } \\
\text { Farbdarstellung } \\
\text { analog offizieller } \\
\text { Gefahrenzonen }\end{array}$ & $\begin{array}{l}\text { - Zu komplex, } \\
\text { Kartenlesbarkeit } \\
\text { eingeschränkt } \\
\text { - Je nach Themen- } \\
\text { auswahl sehr } \\
\text { unübersichtlich }\end{array}$ & $\begin{array}{l}\text { - Übersichtlich, } \\
\text { Lesbarkeit gut } \\
\text { - Farbwahl } \\
\text { ungünstig (keine } \\
\text { Assoziation zu } \\
\text { Wasser) }\end{array}$ & $\begin{array}{l}\text { - Lesbarkeit gut, } \\
\text { Legende versteckt } \\
\text { - Farbwahl negativ } \\
\text { - Historische Hoch- } \\
\text { wasserbilder } \\
\text { und «Eyecatcher» }\end{array}$ \\
\hline Anwendbarkeit & $\begin{array}{l}\text { Hochwasserthema } \\
\text { nur eines unter } \\
\text { vielen räumlichen } \\
\text { Themen } \\
\text { - Für GIS-Laien } \\
\text { schwer nutzbar, } \\
\text { relativiert durch } \\
\text { Standardanzeigen } \\
\text { - Suchfunktionen } \\
\text { eingeschränkt }\end{array}$ & $\begin{array}{l}\text { - Für GIS-Laien } \\
\text { schwer nutzbar } \\
\text { - Eher für gewohnte } \\
\text { GIS-Nutzer } \\
\text { geeignet } \\
\text { - Funktionen/Links } \\
\text { z.T. langsam/gar } \\
\text { nicht nutzbar } \\
\text { - Unzureichend } \\
\text { verlinkt }\end{array}$ & $\begin{array}{l}\text { - Nach kurzer } \\
\text { Einfindung auch } \\
\text { für Laien gut } \\
\text { nutzbar } \\
\text { - Für Fachanwender } \\
\text { zu wenig } \\
\text { Angebote } \\
\text { - Unzureichend } \\
\text { verlinkt }\end{array}$ & $\begin{array}{l}\text { - Nach kurzer } \\
\text { Einfindung auch } \\
\text { für Laien gut } \\
\text { nutzbar } \\
\text { - Für Fachanwender } \\
\text { zu wenig } \\
\text { Angebote }\end{array}$ \\
\hline $\begin{array}{l}\text { Systemanfor- } \\
\text { derungen, Benutzer- } \\
\text { hinweise }\end{array}$ & - Vorhanden & $\begin{array}{l}\text { - z.T. lange } \\
\text { Ladezeit }\end{array}$ & $\begin{array}{l}\text { - Java erforderlich } \\
\text { - z.T. lange } \\
\text { Ladezeit }\end{array}$ & $\begin{array}{l}\text { - Vorhanden, mit } \\
\text { jedem Browser } \\
\text { abrufbar }\end{array}$ \\
\hline
\end{tabular}

Tab. 4: Experten-Evaluation der Internet-Kartendienste und Web-GIS: multimedial-funktionale Dimension Expert evaluation of web mapping services and WebGIS: multimedia and functionality

Evaluation par des experts des services cartographiques web et Web-SIG: multimédia et fonctionnalité

Quelle: eigene Darstellung 


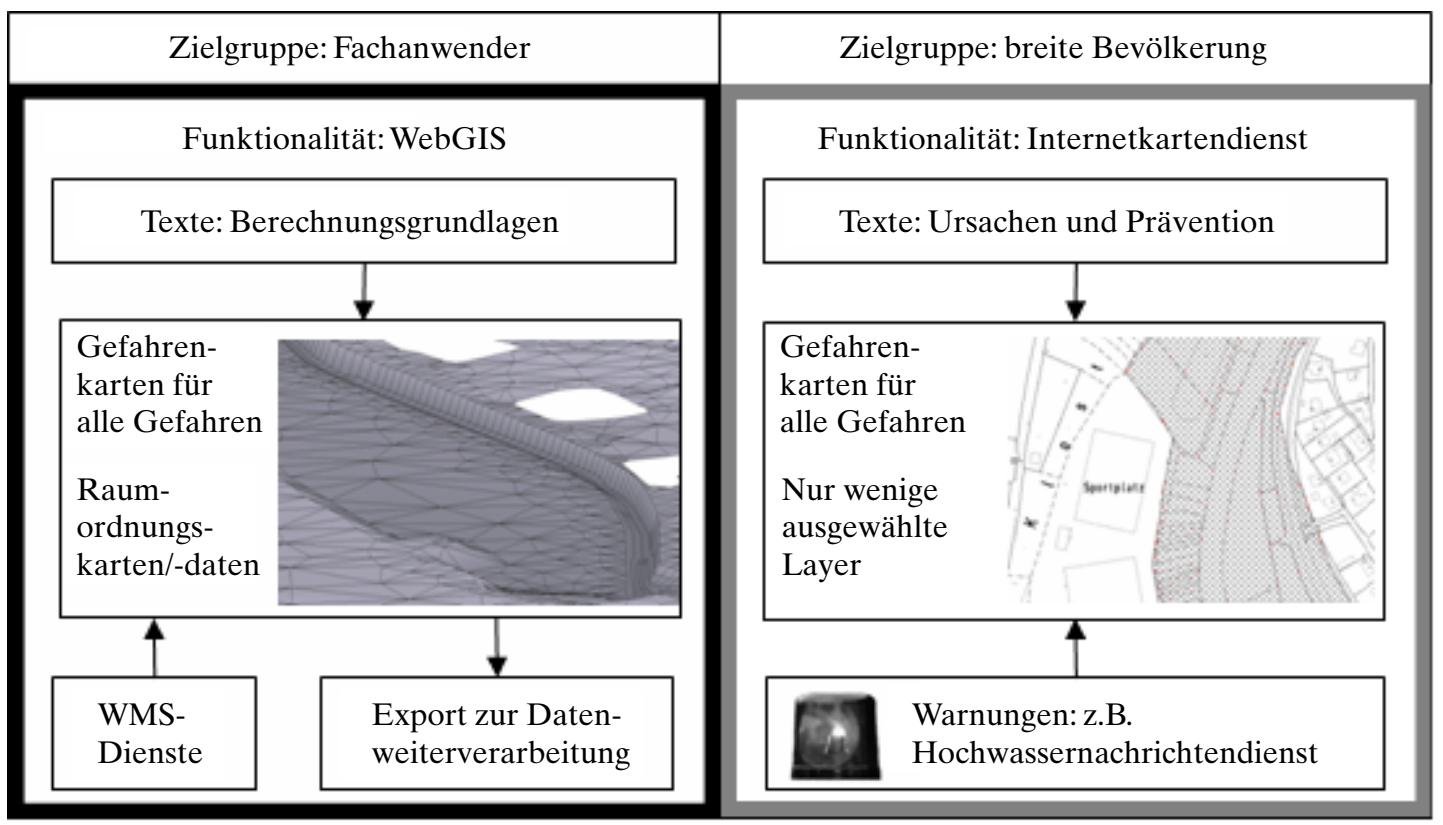

Abb. 1: Aufbau und Inhalte von Informationsdiensten für Fachanwender und für die breite Bevölkerung Design and content of information services for experts and the population Structure et contenu des services d'information pour experts et pour la population Quelle: eigene Darstellung

können zu negativen Online-Erfahrungen beitragen (DrimMel \& Riegl 2007).

Als Handlungsempfehlung kann eine Trennung von Angeboten für die Zielgruppen Fachanwender und breite Bevölkerung formuliert werden mit den entsprechenden Komplexitätsgraden. Die zentralen Bestandteile für diese Nutzergruppen sind in Abb. 1 gegenübergestellt. Bei der Aufbereitung von kartographischen Online-Hochwasser-Informationen für die Bevölkerung spielt die ansprechende und Interesse weckende optische Gestaltung eine wesentlich wichtigere Rolle als bei Expertenangeboten. Je ansprechender und anwendungsfreundlicher die Angebote konzipiert werden, desto attraktiver ist die Nutzung derartiger Informationsportale zu Hochwassergefahren und desto geringer ist die Gefahr der negativen Online-Erfahrung. Damit ebenfalls die Zielgruppe der Kinder oder Jugendlichen erreicht wird, sollten z.B. Unterrichtsmaterial für Lehrer oder spielerische Elemente angeboten werden.

Bei allen Kartendarstellungen, damit auch bei kartographischen Informationen im Internet, ist der «kartographische Analphabetismus» problematisch. Nutzer, die sich auf Offline-Kartenversionen wie Stadtplänen nicht orientieren können, können dies auch online nicht. Hierfür stellt die 3D-Darstellung eine mögliche Lösungsstrategie dar (DrImMel \& RIEGL 2007), die von den evaluierten Diensten bisher noch nicht umgesetzt wird.

Ein Kartendienst oder GIS sollte als ein Modul in ein komplexes Hochwasser-Informations-Portal eingebunden sein, in dem auch andere Angebote wie etwa aktuelle Pegelstände, Informationen zu Ursachen, Schutzeinrichtungen und Vorsorge abrufbar sein sollten. Für die Bevölkerung in hochwassergefährdeten Gebieten ist zudem die Verknüpfung mit EchtzeitInformationen wie aktuellen Messwerten und Warnstufen über eine Verbindung mit Hochwassernachrichtendiensten von großem Interesse, ebenso wie eine Verknüpfung zwischen Pegelständen und zugehörigen Überschwemmungsgebieten. Dies wird lokal bereits in der Hochwassergefahrenkarte Köln oder auch in einem neu entwickelten System in Australien erfolgreich als Online-Visualisierung umgesetzt (BASIC et al. 2005). Webcams an markanten, hochwassergefährdeten Orten, in der Karte eingezeichnet und per Klick ansteuerbar, können während eines Hochwassers als 
Visualisierung der Gefahr dienen. Bei Verwendung von Fachbegriffen wie etwa HQ 100 wird es für essentiell erachtet, in solch einem «Hochwasser-Portal» ausführliche und allgemein verständliche Erklärungen, z.B. in Form eines Glossars, zu geben.

Die Mindestinformationen, die ein Kartendienst bzw. Web-GIS enthalten sollte, sind Abflüsse für verschiedene Hochwasserereignisse mit Darstellung der entsprechenden Wassertiefen, Begriffbestimmungen und Übersichtskarten zur einfachen Navigation. $\mathrm{Zu}$ empfehlen wäre des Weiteren der Einsatz von Bildern tatsächlicher historischer Hochwasserereignisse, um einen realen Bezug herzustellen und das Risikobewusstsein in der Bevölkerung zu erhöhen.

Fachanwender benötigen dagegen eher Informationen über Berechnungsgrundlagen der dargestellten Modellierungsergebnisse, Verschneidungsmöglichkeiten mit anderen planungsrelevanten Kartenwerken und Daten sowie Exportmöglichkeiten zur Weiterverarbeitung in anderen Systemen. Bei Fachanwendern spielt zudem die optische Gestaltung gegenüber einer ausgeweiteten Funktionalität eine untergeordnete Rolle.

\section{Fazit und Ausblick}

Die derzeit im Internet verfügbaren geographischen Informationsangebote zur Kommunikation von Hochwasserrisiken teilen sich in komplexe GIS-Lösungen und einfach gehaltene Kartendienste. Um nutzerangepasste Internetangebote bereitzustellen, ist eine Trennung von Angeboten für Experten und für die breite Bevölkerung sinnvoll. Für Fachanwender sollten die Internetangebote weit reichende GIS-Funktionalität mit Daten-Downloads zur Weiterverwendung enthalten. Für die Bevölkerung sollten die Angebote optisch ansprechend gestaltet, übersichtlich, leicht verständlich und nur mit den notwendigsten Funktionen ausgestattet sein, um eine Überforderung zu vermeiden. Verknüpfungen mit Echtzeit-Informationen und 3DDarstellungen können die Angebote abrunden.

Im Hinblick auf die rasanten technischen Entwicklungen und auf die stetig zunehmende Nutzung von mobilen Computern und internet- sowie GPS-fähigen Mobiltelefonen müssen sich die Anbieter von Internetkartendiensten und Web-GIS-Lösungen auch diesen neuen Herausforderungen stellen und darauf angepasste Informationsangebote bereithalten. Ebenso wichtig erscheint zukünftig im Web 2.0 das Angebot, derartige Informationsdienste selbst aktiv mitgestalten zu können und vergleichbar zu Google Earth personalisierte Kartenwerke erstellen und nutzen zu können.

\section{Literatur}

Arbeitsgemeinschaft Online Forschung (AGOF) E.V. (2007): Berichtsband - Teil $1 \mathrm{zu}$ Internet Facts 2007-II. - http://www.agof.de/studie.353.html 13.11.07. Alexander, J.E. \& M.A. TAte (1999): Web wisdom: how to evaluate and create information quality on the Web. - Mahwah, N.J.: Lawrence Erlbaum.

Basic, F., Handmer, J. \& W. Cartwright (2005): Communicating flood risks to the public through visualisation in scientific computing. - In: Proceedings of the $22^{\text {nd }}$ International Cartographic Conference, 11-16 July, in Coruña, Spain: 2790-2796.

BUNDESAMT FÜR STATISTIK (BFS) (2007): Internetnutzung. - http://www.bfs.admin.ch/bfs/portal/de/index/ themen/16/04/key/approche_globale.indicator.30106. 301.html?open=1\#1 13.11.07.

BundesministeriUm FÜR UMWElt, NATURSCHUTZ UND REAKTORSICHERHEIT (BMU) (2007): Pressemitteilung Nr. 117/07 vom 26.04.2007.

Deutsche Bundesstiftung Umwelt (DBU) (2005): Materialien zur Internetstudie «Internetanwendungen im Natur- und Umweltschutz». - www.dbu.de/621 13.11.07.

Drimmel, C. \& A. RiEgL (2007): Geo-tagging - community-Bildung durch eine neue Publikationsform im Internet. - = Netidee 939: 1-84.

EUROSTAT (2007): Informationsgesellschaft. - http:// epp.eurostat.ec.europa.eu/ 13.11.07.

Hochwassergefahrenkarten Köln. - www.hw-karten. de/koeln/ 10.12.2007.

InFORMATIONSDiENST Alpine NATURgeFAHREN (IAN) (2007). - http://www.lfu.bayern.de/wasser/fachinformationen/ian/index.htm 10.12.2007.

INFORMATIONSDIENST ÜBERSCHWEMMUNGSGEFÄHRDETE GEBIETE IN BAYERN (IÜG) (2007). - www.bayern.de/ LFW/iug/ 10.12.2007.

Kärnten Atlas (2007). - Herausgegeben vom Bundesamt für Eich- und Vermessungswesen (BEV); http:// www.verwaltung.ktn.gv.at/19948_DE 10.12.2007.

Möller, M. (2006): Die Geo-Komponente in der Informationsgesellschaft? Auf dem Weg zur GeoKommunikation. - In: Kartographische Nachrichten 5: 239-243.

Nationale Plattform Naturgefahren (PLANAT) (2004): Hochwassergefahren in der Schweiz: Risikobewusstsein in der Bevölkerung und die Implikationen für eine erfolgreiche Risikokommunikation. - Biel: PLANAT.

Riemer, K. \& C. Müller-Lankenau (2005): Web-Evaluation: Einführung in das Internet-Qualitätsmanagement. - = Internetökonomie und Hybridität Nr. 21, Münster:1-43.

Scharl, A. \& K. Tochtermann (Hrsg.) (2007): The geospatial web - how geobrowsers, social software and the Web 2.0 are shaping the network society. - London: Springer.

Schrems, J. (1998): Die Sicherheitskompetenz der 
Forstwirtschaft in Österreich. - Dissertation am Institut für Sozioökonomik der Forst- und Holzwirtschaft an der Universität für Bodenkultur, Wien.

Siegrist, M. \& H. GuTscher (2006): Flooding risks: a comparison of lay people's perceptions and expert's assessments in Switzerland. - In: Risk analysis 26: 971979.

SMITH, A. (1997): Testing the surf: criteria for evaluating internet information resources. - In: The publicaccess computer review 8, 3: o. S.

Van Eimeren, B. \& B. Frees (2007): Internetnutzung zwischen Pragmatismus und YouTube-Euphorie. - In: Media Perspektiven 8: 362-378.

WAGNER, K. (2004): Naturgefahrenbewusstsein und -kommunikation am Beispiel von Sturzfluten und Rutschungen in vier Gemeinden des bayerischen Alpenraums. - Dissertation an der Studienfakultät Forstwissenschaft und Ressourcenmanagement der Technischen Universität München.

Wottawa, H. \& H. Thierau (2003): Lehrbuch Evaluation. - 3. Auflage, Bern: Huber.

\section{Zusammenfassung: Experten-Evaluation von Web- GIS-Lösungen und Internetkartendiensten zur Kom- munikation von Hochwasserinformationen}

Behörden gehen im Zeitalter der neuen Medien zunehmend dazu über, Hochwasserinformationen über das Internet zu vermitteln. Dieser Beitrag umfasst einen typisierenden Überblick über aktuell im Internet zur Hochwasserinformation verfügbare Kartendienste und Web-GIS sowie eine ExpertenEvaluation ausgewählter Angebote. Die analysierten Angebote unterscheiden sich stark in Funktionalität und Inhalt. Es handelt sich sowohl um für Nutzer aus der Bevölkerung zu komplexe GIS-Lösungen, als auch um einfache Kartendienste, die jedoch für Fachanwender ungenügende Funktionalität aufweisen. Eigene Interaktionsmöglichkeiten bieten sich für die Nutzer derzeit kaum. Als Empfehlungen werden Ausweitung der Interaktivität, zielgruppenspezifische Ausrichtung der Angebote sowie Erweiterungen um Echtzeitinformationen formuliert.

Schlüsselwörter: Risikokommunikation, Hochwasser, Öffentlichkeit, Web-GIS, Evaluation

\footnotetext{
Abstract: Expert evaluation of WebGIS and web mapping services for publicising flood information

Authorities are increasingly making flood information public via the internet. This article gives an overview of current web mapping services and WebGIS on flood risks and evaluates the content and usabil-
}

ity of four web sites. Currently, the palette ranges from GIS solutions that are too complex for laymen to very simple web mapping services providing insufficient information for experts. Additionally, the present services hardly offer interaction possibilities. It is recommended that interactivity be extended to include real time information and to adjust the services to the needs of different target groups.

Keywords: risk communication, flooding, publicity, WebGIS, evaluation

Résumé: Expertise de solutions Web-SIG et de services cartographiques Internet servant à la communication sur les risques de crues

A l'ère des nouveaux médias, les autorités recourent de plus en plus à Internet pour transmettre des informations relatives aux crues. L'objectif de cette contribution est d'offrir une vue d'ensemble des services cartographiques et SIG disponibles sur Internet qui transmettent des informations relatives aux crues et de présenter leur évaluation par des experts. Les offres analysées diffèrent fortement selon leur fonctionnalité et leur contenu. On relève des solutions SIG trop complexes pour les amateurs ainsi que des services cartographiques qui manquent de fonctionnalité pour l'utilisateur spécialisé. A l'heure actuelle, très peu de possibilités de réelle interaction sont offertes aux utilisateurs; c'est pourquoi l'article recommande d'approfondir l'interactivité, de cibler l'offre sur des clientèles spécifiques et d'encourager la fourniture d'informations en temps réel.

Mots-clés: communication relative aux risques, crues, public, Web-SIG, évaluation

Dipl.-Geogr. Maria Hagemeier-Klose, Dr. Klaus Wagner, Lehrstuhl für Wald- und Umweltpolitik, Technische Universität München, Am Hochanger 13, D-85354 Freising, Deutschland.

e-mail:

hagemeier@forst.wzw.tum.de

wagner@forst.wzw.tum.de

\section{Manuskripteingang/received/manuscrit entré le} 14.1.2008

Annahme zum Druck/accepted for publication/accepté pour l'impression: 5.6.2008 